\title{
PERFORATION OF THE TRACHEA AND BRONCHUS BY THE BRONCHOSCOPE
}

\author{
BY \\ W. GORDON \\ From the Regional Thoracic Surgery Centre, Shotley Bridge Hospital, \\ Newcastle-upon-Tyne
}

(RECEIVED FOR PUBLICATION AUGUST 26, 1950)

A case of perforation of the trachea by the bronchoscope prompted a study of the records of this clinic, and it was found that in approximately 15,000 bronchoscopies performed during the last 13 years only one similar case, a perforation of the bronchus, had occurred. These two cases are now recorded.

In the literature of the past 20 years there appears to be no record of perforation of the intrathoracic portion of the trachea or the bronchi by the bronchoscope nor any reference to such an accident having occurred previously. Possibly, unrecorded cases have occurred. Describing a patient with mediastinal emphysema following bronchoscopy, Jones (1945) assumed damage at some point in the tracheo-bronchial tree, but the site and nature of the lesion were not visualized.

In the following cases there was bronchoscopic and radiographic or clinical evidence of a perforated trachea in one patient and a perforated bronchus in the other.

\section{CASE REPORTS}

Case 1.-On April 26, 1950, A. H., a man aged 49 years, was under investigation for suspected bronchial carcinoma. He had received routine medication with omnopon gr. 1/3 and hyoscine gr. 1/150, had sucked a 1 gr. tablet of amethocaine, and had had a small wad of wool soaked in amethocaine solution pressed into each pyriform fossa. The patient was very co-operative, and a bronchoscope was passed without difficulty between normal vocal cords into the trachea. The trachea and main carina were normal, and the right main bronchus was about to be examined when the patient gave a single, but powerful, cough. The operator withdrew his eye from the bronchoscope for a moment, but on looking down again a tear about $1 \mathrm{~cm}$. long was seen in the right wall of the trachea immediately above and behind the origin of the right main bronchus. The tear appeared to be at the junction of the cartilaginous and muscular portions of the trachea. The examination of the bronchial tree was completed, the patient had a radiograph taken, and then returned to the ward. Already subcutaneous emphysema was present in the neck and face. The patient did not complain of pain or discomfort, although his sensorium may have been dulled by pre-medication, and there were no signs of respiratory or circulatory embarrassment. Under local analgesia a $3 \mathrm{~cm}$. transverse incision was 


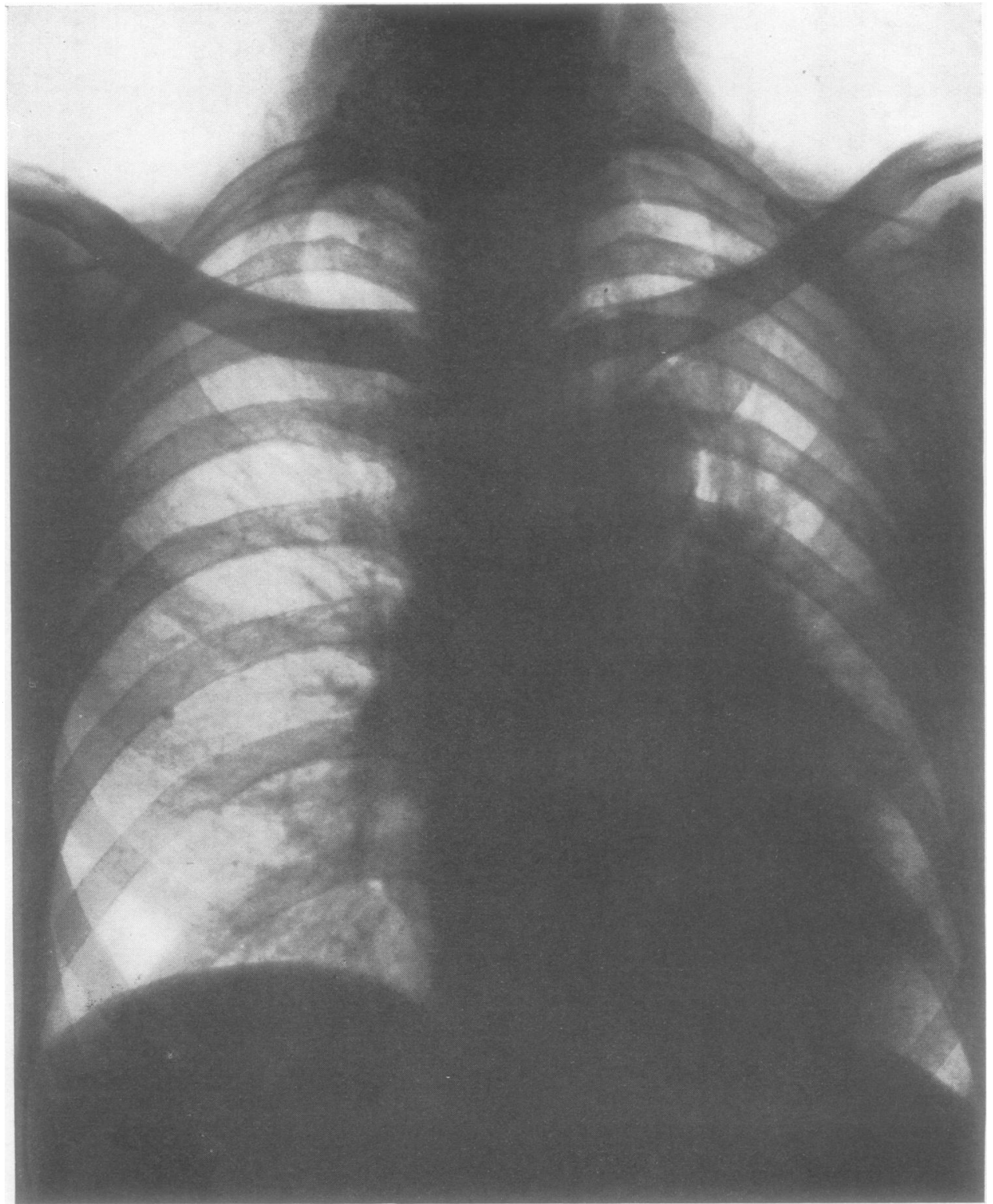

FIG. 1.-Radiograph taken soon after bronchoscopy (Case 1). Air can be seen outlining the aortic arch, in the left side of the superior mediastinum, and in the neck.

made through the skin of the neck just above the upper border of the manubrium sterni and carried down to the trachea. Air bubbled out of the wound during dissection. A small dry gauze dressing was lightly applied to the wound and the patient was given an intramuscular injection of 500,000 units of penicillin in an effort to prevent mediastinitis and a cough sedative was prescribed. 
The radiograph (Fig. 1), taken immediately after bronchoscopy, showed the aortic arch clearly outlined by a crescent of air, and air could be seen in the left side of the mediastinum and in the neck.

Radiographs taken daily for the next three days showed decreasing amounts of air in the mediastinum and neck. The film seven days later (Fig. 2) was similar to

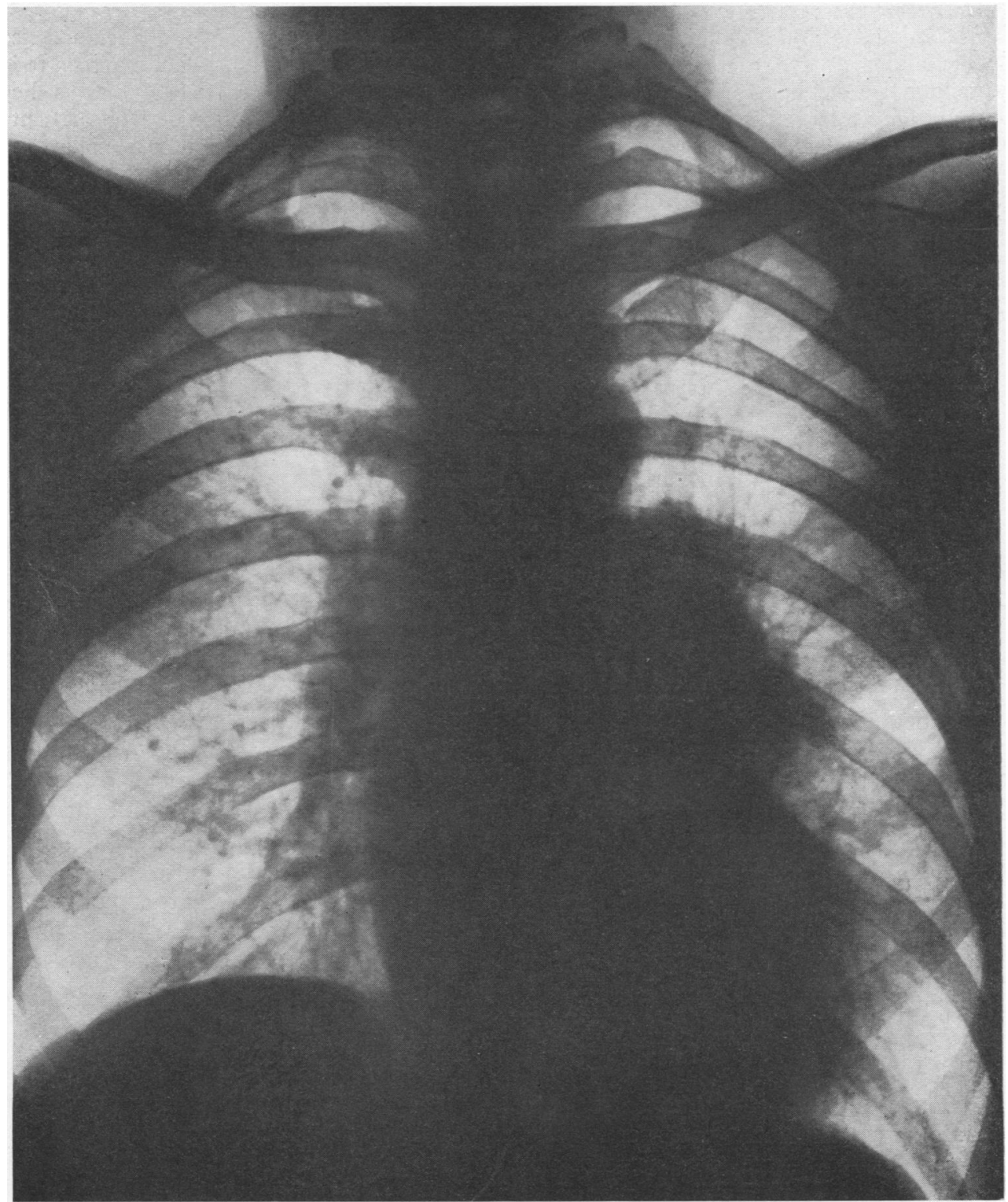

FIG. 2.- Radiograph taken seven days after the film shown in Fig. 1. The appearances are the same as those before bronchoscopy. The shadow of the bronchial carcinoma can be seen in the left hilar region. 
the film before bronchoscopy. During that week the patient received 500,000 units of penicillin every six hours and his temperature remained within normal limits. The neck incision healed in a few days. Convalescence from the tracheal injury was uneventful, and 15 days later bronchoscopy revealed a normal trachea. This bronchoscopy preceded a thoracotomy at which an irremovable bronchial carcinoma was found.

Case 2.-H. B., a man aged 45 years, was under investigation for haemoptysis which later proved to be due to tuberculosis. On September 24, 1940, he received preliminary medication with omnopon gr. $1 / 3$ and hyoscine gr. $1 / 100$, but as he would not tolerate local analgesia pentothal anaesthesia was induced. The patient tended to gag when the bronchoscope was passed. A slight abrasion was made on the left main bronchus and another on the left upper lobe bronchus near its origin. The second injury resulted in profuse bleeding and further examination was not made. The following day the patient complained of a sore throat and subcutaneous emphysema was present, first in the neck and later also on the face, chest, and abdomen. No decompressing incision was made. The patient received one tablet of sulphapyridine every four hours for the next four days. During this time his temperature remained normal and the subcutaneous emphysema gradually disappeared. Bronchoscopic appearances were normal 27 days later.

\section{Discussion}

Air in the mediastinum of both patients escaped quite readily into the subcutaneous tissues of the neck so that mediastinal structures were not compressed. The incision in the neck of A.H. (Case 1) prevented the discomfort of more generalized subcutaneous emphysema ; probably there was no dramatic decompression of the mediastinum. This easy escape of air from the mediastinum to the subcutaneous tissues is common. Hamman (1945) points out that subcutaneous emphysema in the neck may be the only, yet convincing, evidence of mediastinal emphysema but that mediastinal emphysema does not always lead to subcutaneous emphysema. The latter, of course, can occur without mediastinal emphysema. Air may have travelled from the mediastinum into the neck along the great vessels arising from the aorta. Hammond (1944), describing a case of mediastinal and subcutaneous emphysema in an 18-months-old boy, thought that air had passed out of the mediastinum "by way of blood vessel sheaths." There is evidence that air may travel to, and possibly from, the mediastinum in the perivascular plane (Kelman, 1919; Macklin, 1939). However, at some point, either intrathoracic or cervical, the deep fascia in these two patients did not exist in definite sheaths or, if it did, was penetrated easily by air under pressure less than that which would cause vascular or respiratory embarrassment.

\section{SUMMARY}

Perforation of the trachea or bronchi by the bronchoscope is rare. Two cases of mediastinal emphysema following bronchoscopy are reported. In one patient this was due to a perforation of the trachea and in the other to a perforation of a bronchus. One patient was treated by a suprasternal incision of the soft tissues and penicillin; the other received sulphapyridine only. Both made an uneventful recovery. 
The cases are recorded by kind permission of the Director of the Regional Thoracic Surgery Centre, Mr. George A. Mason.

\section{REFERENCES}

Hamman, L. (1945). J. Amer. med. Ass., 128, 1.

Hammond, A. E. (1944). Ann. Otol., etc., St. Louis, 53, 829.

Jones, R. (1945). Brit. med. J., 2, 530.

Kelman, S. R. (1919). Arch. intern. Mcd., 24, 332.

Macklin, C. C. (1939). Jbid., 64, 913. 